August 11, 2021 12:15 WSPC/INSTRUCTION FILE vanoerscospa07

Modern Physics Letters A

(C) World Scientific Publishing Company

\title{
FROM HADRONIC PARITY VIOLATION TO PARITY-VIOLATING ELECTRON SCATTERING AND TESTS OF THE STANDARD MODEL *
}

\author{
WILLEM T. H. VAN OERS \\ Department of Physics and Astronomy, University of Manitoba, \\ Winnipeg, MB, Canada R3T 2N2 \\ and \\ TRIUMF, 4004 Wesbrook Mall, Vancouver, BC, Canada V6T 2A3 ${ }^{\dagger}$
}

Received (Day Month Year)

Revised (Day Month Year)

\begin{abstract}
Searches for parity violation in hadronic systems started soon after the evidence for parity violation in $\beta$-decay of ${ }^{60} \mathrm{Co}$ was presented by Madame Chien-Shiung Wu and in $\pi$ and $\mu$ decay by Leon Lederman in 1957. The early searches for parity violation in hadronic systems did not reach the sensitivity required and only after technological advances in later years was parity violation unambiguously established. Within the meson-exchange description of the strong interaction, theory and experiment meet in a set of seven weak meson-nucleon coupling constants. Even today, after almost five decades, the determination of the seven weak meson-nucleon couplings is incomplete. Parity violation in nuclear systems is rather complex due to the intricacies of QCD. More straight forward in terms of interpretation are measurements of the proton-proton parity-violating analyzing power (normalized differences in scattering yields for positive and negative helicity incident beams), for which there exist three precision experiments (at 13.6, at 45, and $221 \mathrm{MeV}$ ). To-date, there are better possibilities for theoretical interpretation using effective field theory approaches.

The situation with regard to the measurement of the parity-violating analyzing power or asymmetry in polarized electron scattering is quite different. Although the original measurements were intended to determine the electro-weak mixing angle, with the current knowledge of the electro-weak interaction and the great precision with which electro-weak radiative corrections can be calculated, the emphasis has been to study the structure of the nucleon, and in particular the strangeness content of the nucleon. A whole series of experiments (the SAMPLE experiment at MIT-Bates, the G0 experiment and HAPPEX experiments at Jefferson Laboratory (JLab), and the PVA4 experiment at MAMI) have indicated that the strange quark contributions to the charge and magnetization distributions of the nucleon are tiny. These measurements if extrapolated to zero degrees and zero momentum transfer have also provided a factor five improvement in the knowledge of the neutral weak couplings to the quarks.

Choosing appropriate kinematics in parity-violating electron-proton scattering permits nucleon structure effects on the measured analyzing power to be precisely controlled. Consequently, a precise measurement of the 'running' of $\sin ^{2} \theta_{W}$ or the electro-weak
\end{abstract}

\footnotetext{
*Work supported in part by NSERC (Canada) and TRIUMF
}

†email: vanoers@triumf.ca 


\begin{abstract}
mixing angle has become within reach. The $Q_{\text {weak }}^{p}$ experiment at Jefferson Laboratory is to measure this quantity to a precision of about $4 \%$. This will either establish conformity with the Standard Model of quarks and leptons or point to New Physics as the Standard Model must be encompassed in a more general theory required, for instance, by a convergence of the three couplings (strong, electromagnetic, and weak) to a common value at the GUT scale.

The upgrade of CEBAF at Jefferson Laboratory to $12 \mathrm{GeV}$, will allow a new measurement of $\sin ^{2} \theta_{W}$ in parity-violating electron-electron scattering with an improved precision to the current better measurement (the SLAC E158 experiment) of the 'running' of $\sin ^{2} \theta_{W}$ away from the $Z^{0}$ pole. Preliminary design studies of such an experiment show that a precision comparable to the most precise individual measurements at the $Z^{0}$ pole (to about $\pm 0.00025)$ can be reached. The result of this experiment will be rather complementary to the $Q_{w e a k}^{p}$ experiment in terms of sensitivity to New Physics.
\end{abstract}

Keywords: Parity Violation

PACS Nos.: 11.30.En; 12.60.-i; 1420.Dh

\title{
1. Hadronic Parity Violation
}

Manifestations of the weak interaction of quarks have been searched for since Madame Chien-Shiung Wu presented the evidence for parity violation in $\beta$-decay of ${ }^{60} \mathrm{Co}$ and Leon Lederman for parity violation in $\pi$ and $\mu$ decay. For instance, Neil Tanner shortly thereafter, studied the $340 \mathrm{keV}$ resonance in the ${ }^{19} F(p, \alpha)$ reaction to the ${ }^{16} \mathrm{O}$ ground state forbidden by angular momentum and parity conservation.[1] The hadronic weak interaction is studied by observing non-leptonic flavor changing decays of mesons and baryons and by measuring observables that conserve flavor but that violate reflection symmetry of the strong and electromagnetic interactions. One approach that has been pursued is the study of parity forbidden transitions between nuclear states in particular in the light nuclei. It was realized that two accidental aspects of nuclear structure in certain nuclei could amplify the expected effects of parity violation by several orders of magnitude beyond the nominal $O\left(10^{-7}\right)$. The amplification arises from the near-degeneracy of opposite parity states that are mixed by the hadronic weak interaction and from the interference of an otherwise by parity conservation forbidden transition amplitude with a much stronger parity allowed transition amplitude. Although the weak quark-quark interaction has been well established its appearance in nuclear systems is clouded by effects of nuclear structure and by the dynamics of quantum chromodynamics (QCD) in the non-perturbative regime. Within the meson exchange description of the strong interaction, theory and experiment meet in a set of seven weak meson-nucleon coupling constants as defined in the seminal paper by Desplanques, Donoghue, and Holstein (DDH). [2] It is assumed that the parity violating $\mathrm{N}-\mathrm{N}$ interaction is governed by the exchange of the pion and the two lightest vector mesons. The seven weak meson nucleon coupling constants are designated $h_{\pi}^{1}, h_{\rho}^{0,1,2}, h_{\rho}^{1^{\prime}}$, and $h_{\omega}^{0,1}$, where the superscript indicates the isospin and the subscript the exchanged meson. Of these $h_{\rho}^{1^{\prime}}$ was omitted from further consideration due to the difficulty calculating it. Desplanques, Donoghue, and Holstein provided 'reasonable ranges' and 'best values' for the weak 
Table 1. Theoretical 'reasonable ranges' (second column) and 'best values' columns (three to five) for the parity violating meson-nucleon coupling constants from Desplanques, Donoghue, and Holstein, from Dubovic and Zenkin, and from Feldman et al.. All values are given in units of $g_{\pi}=3.8 \times 10^{-8} \approx G_{F} F_{\pi}^{2} /(2 \sqrt{2})$.

\begin{tabular}{lcccr}
\hline Coupling constant & DDH range & DDH best value & DZ & FCDH \\
\hline$h_{\pi}^{1}$ & $0 \rightarrow-30$ & +12 & +3 & +7 \\
$h_{\rho}^{0}$ & $30 \rightarrow-81$ & -30 & -22 & -10 \\
$h_{\rho}^{1}$ & $-1 \rightarrow 0$ & -0.5 & +1 & -1 \\
$h_{\rho}^{2}$ & $-20 \rightarrow-29$ & -25 & -18 & -18 \\
$h_{\omega}^{0}$ & $15 \rightarrow-27$ & -5 & -10 & -13 \\
$h_{\omega}^{1}$ & $-5 \rightarrow-2$ & -3 & -6 & -6 \\
\hline
\end{tabular}

meson nucleon couplings constants. These are given in Table 1 together with values for the latter from two similar theoretical approaches (Dubovic and Zenkin [3], and Feldman et al. [4]).

The experimental results from the nuclear parity violating measurements have been analyzed using the framework of Desplanques, Donoghue, and Holstein leading to constraints on combinations of the weak meson nucleon coupling constants. There is a measure of agreement with the theoretical 'reasonable ranges' although also experimentally the allowed ranges of values are large. Note that there exist two experimental inconsistencies as depicted in Fig. 1: the value of $h_{\pi}^{1}$ deduced from the $\gamma$-decays of ${ }^{18} \mathrm{~F}$ is consistent with zero while the result deduced from the anapole moment of ${ }^{133} \mathrm{Cs}$ indicates a difference from zero with several standard deviations. The other inconsistency is possibly in the results deduced from the anapole moments of ${ }^{205} \mathrm{Tl}$ and ${ }^{133} \mathrm{Cs}$. More straightforward in terms of interpretation are the measurements of the proton-proton parity violating analyzing powers or asymmetries (the normalized differences in scattering yields for positive and negative helicity incident beams) for which there exist three precision experiments (from the University of Bonn at 13.6 MeV [6], from PSI at $45 \mathrm{MeV}$ [7], and from TRIUMF at $221 \mathrm{MeV}$ [8]). These experiments required unprecedented precision in controlling systematic errors and in particular those due to helicity correlated false asymmetries. The parity violating analyzing powers are compared to more recent theoretical calculations in the framework of weak meson-nucleon couplings in Fig. 2. (see [8]) Carlson et al. [9] deduced the constraints on the two combinations of weak meson-nucleon coupling constants: $h_{\omega}^{p p}=h_{\omega}^{0}+h_{\omega}^{1}$ and $h_{\rho}^{p p}=\left(h_{\rho}^{0}+h_{\rho}^{1}+h_{\rho}^{2} / \sqrt{6}\right)$ from the proton-proton analyzing power data. Clearly even more precise measurements of the proton-proton analyzing powers are required in order to better constrain the weak meson-nucleon coupling constants (see Fig. 3).

The extensive development of chiral perturbation theory and N-N effective field theory and substantial progress in performing lattice QCD calculations have opened up new approaches of studying the hadronic weak interaction. For a recent review see Ref. 10. In principle it is now possible to make QCD based predictions for weak hadronic interaction phenomena. On the experimental side there are several promis- 


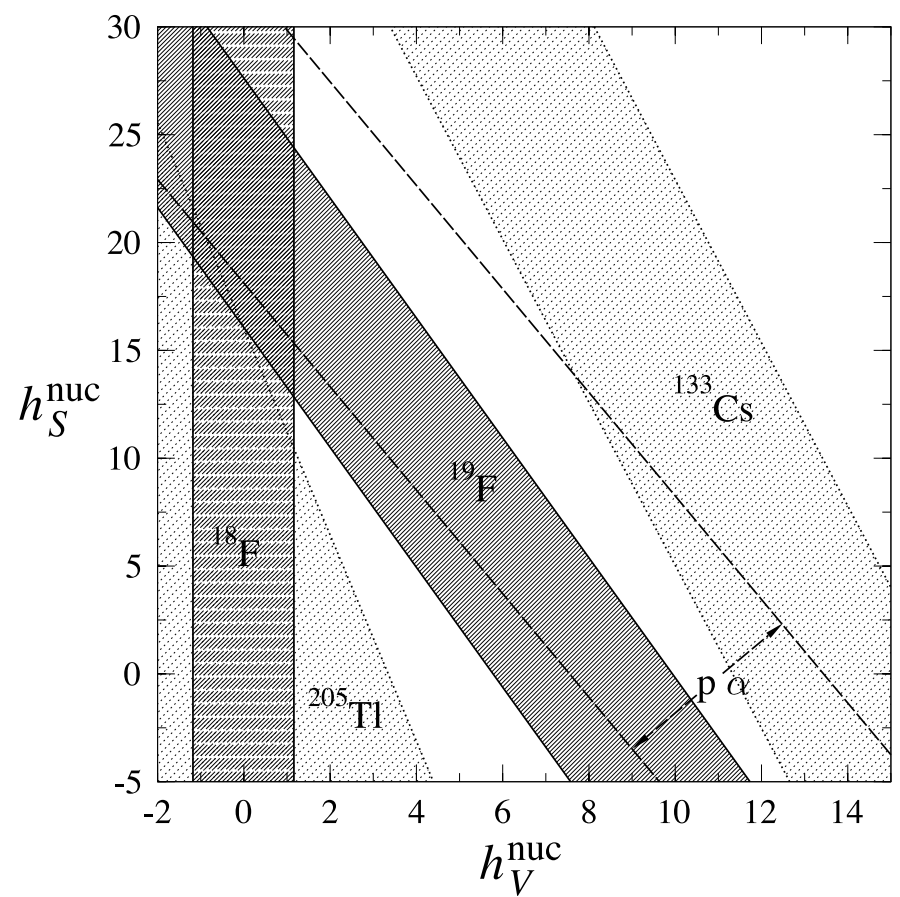

Fig. 1. Constraints on effective DDH weak meson-nucleon coupling constants deduced from parity violating observables in light nuclei and anapole moments of heavy atoms (see [5]). The combinations of the weak meson-nucleon coupling constants are: $h_{V}^{n u c}=h_{\pi}^{1}-0.12 h_{\rho}^{1}-0.18 h_{\omega}^{1}$ and $h_{S}^{n u c}=-\left(h_{\rho}^{0}+0.7 h_{\omega}^{0}\right)$

ing precision parity violation measurements in progress. Foremost to be mentioned is the measurement of the parity violating asymmetry in the capture of polarized neutrons on hydrogen (the NPDGAMMA experiment). Others are the parity violating neutron spin rotation in helium and in hydrogen. (see Ref. 10)

\section{Parity Violating Electron Scattering}

The situation with regard to parity violating analyzing power measurements in polarized electron scattering is quite different. Originally, in the low energy regime, these measurements were made to determine the weak mixing angle or $\sin ^{2} \theta_{W}$. Soon thereafter these measurements were overtaken by measurements at the $Z^{0}$ pole at LEP of CERN and SLC of SLAC. Given the current knowledge of the electroweak interaction and the great precision with which electroweak radiative corrections can be calculated, the current round of parity violating electron scattering experiments has as one of its objectives to probe the structure of the nucleon and in particular the contributions of the strange quarks (the quark-antiquark pairs of the sea) to the weak charge and magnetization distributions of the nucleons. With the couplings of both photons and $\mathrm{Z}$ bosons to point-like quarks well defined (see Table 2), it is 


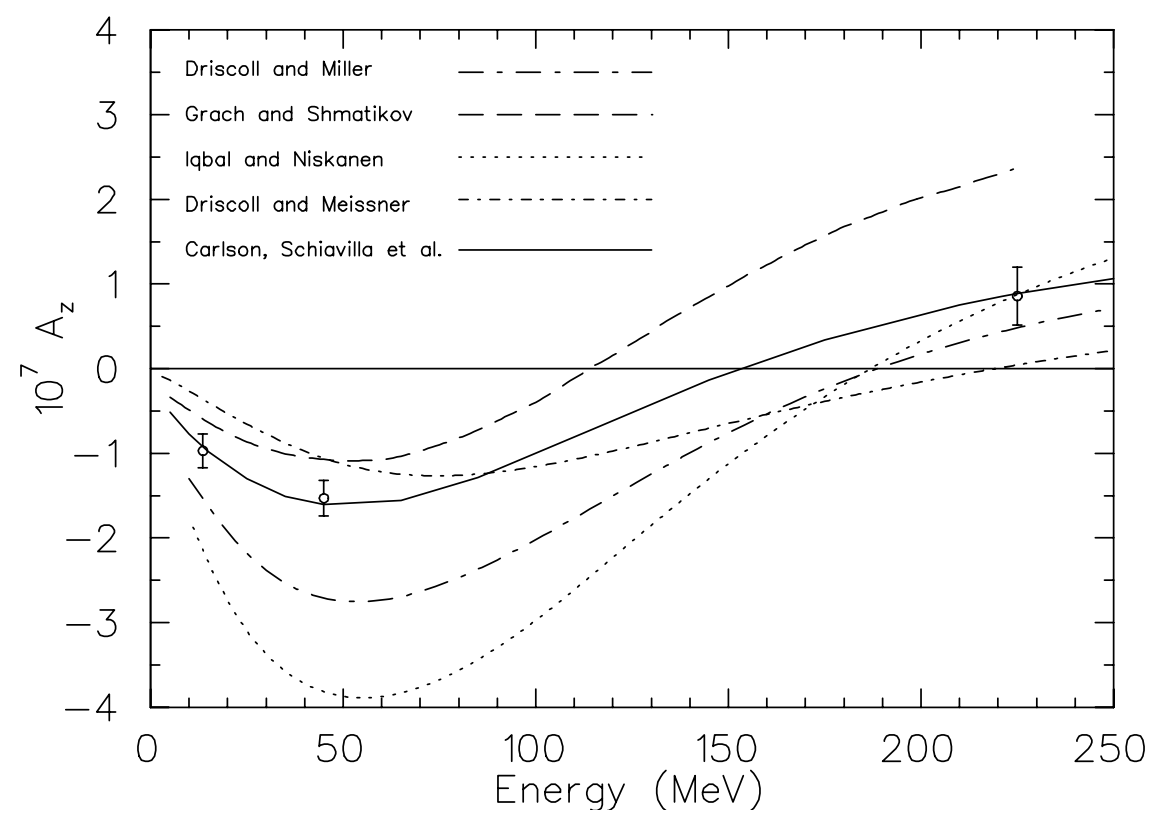

Fig. 2. The three most precise proton-proton parity violation measurements (at 13.6, 45, and $221 \mathrm{MeV}$ ) and theoretical predictions. The solid curve shows the calculation by Carlson et al.[9] in which the weak meson nucleon coupling constants were fitted to the data.

possible to separate the contributions of the various flavors. The electromagnetic and weak charge and magnetic form factors of the proton can be written:

$$
G_{E, M}^{\gamma, Z}=2 q^{u} G_{E, M}^{u}+q^{d} G_{E, M}^{d}+q^{d} G_{E, M}^{s}
$$

Heavier flavor contributions are neglected. Assuming charge symmetry in the quark distributions, one can write an analogous expression for the neutron:

$$
G_{E, M}^{\gamma, Z}{ }^{n}=2 q^{d} G_{E, M}^{d}+q^{u} G_{E, M}^{u}+q^{d} G_{E, M}^{s}
$$

Clearly, in addition to the electromagnetic form factors for the proton and the neutron one needs one further relation, which is provided by the parity violating analyzing power $A_{z}$ :

$$
\begin{aligned}
A_{z} & =(1 / P)\left[N^{+}-N^{-}\right] /\left[N^{+}+N^{-}\right] \\
& =-\left[G_{F} Q^{2} /(4 \sqrt{2} \pi \alpha)\right] \times\left[\epsilon G_{E}^{\gamma} G_{E}^{Z}+\tau G_{M}^{\gamma} G_{M}^{Z}-\left(1-4 \sin ^{2} \theta_{W}\right) \epsilon^{\prime} G_{M}^{\gamma} G_{A}^{e}\right] / D
\end{aligned}
$$

where $N^{+}$and $N^{-}$are the normalized scattering yields and

$$
\begin{aligned}
\tau & =Q^{2} /\left(4 M_{p}^{2}\right), \\
\epsilon & =1 /\left(1+2(1+\tau) \tan ^{2}(\theta / 2)\right), \\
D & =\epsilon\left(G_{E}^{\gamma}\right)^{2}+\tau\left(G_{M}^{\gamma}\right)^{2} \\
\epsilon^{\prime} & =\sqrt{\tau(1+\tau)\left(1-\epsilon^{2}\right)},
\end{aligned}
$$






Fig. 3. Constraints on the weak meson-nucleon coupling constants, combinations $h_{\omega}^{p p}$ and $h_{\rho}^{p p}$ as deduced from the proton-proton parity violating analyzing powers. [9] The figure shows contours of constant total $\chi^{2}=1,2,3,4,5$. The axes scales are in units of $10^{-7}$.

and $Q^{2}$ is the squared four-momentum transfer, $G_{F}$ is the Fermi coupling constant and $\alpha$ is the fine structure constant. The three new form factors $G_{E}^{Z}, G_{M}^{Z}$, and $G_{A}^{e}$ can be determined by measuring parity violating electron elastic scattering from the proton at forward and backward angles, and quasi-elastic scattering from the deuteron at backward angles.

Table 2. Electroweak charge phenomenology. Note the accidental suppression of the weak charge of the proton. The two charges of the proton and the neutron are to a large extent interchanged.

\begin{tabular}{lcr}
\hline & Electromagnetic Charge & Weak Charge \\
\hline$q^{u}$ & $+2 / 3$ & $1-(8 / 3) \sin ^{2} \theta_{W} \approx 1 / 3$ \\
$q^{d}$ & $-1 / 3$ & $-1+(4 / 3) \sin ^{2} \theta_{W} \approx-2 / 3$ \\
$Q^{p}=2 q^{u}+1 q^{d}$ & +1 & $1-4 \sin ^{2} \theta_{W}=0.0716$ \\
$Q^{n}=1 q^{u}+2 q^{d}$ & 0 & -1 \\
\hline
\end{tabular}

Presently there exist published data on a combination $G_{E}^{s}+\eta G_{M}^{S}$, with $\eta$ a coefficient dependent on the four-momentum transfer, from the SAMPLE experi- 


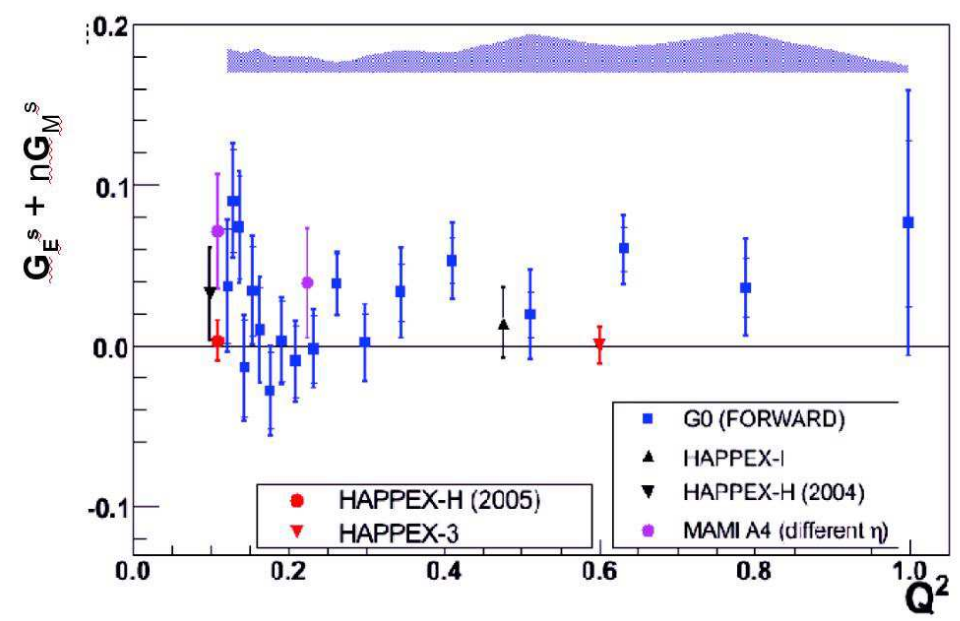

Fig. 4. The combination $G_{E}^{s}+\eta G_{M}^{s}$ as deduced from the G0, HAPPEX, and PVA4 experiments. The blue-gray band indicates the dominant systematic uncertainty in the G0 results.

ment at MIT-Bates [11], the HAPPEX [12] and G0 [13] experiments at JLab, and the PVA4 experiment at MAMI 14 (see Fig. 4). There is consistency between the various experimental results. The data indicate tiny non-zero values for the combination $G_{E}^{s}+\eta G_{M}^{S}$. The backward angle data on hydrogen and deuterium of the G0 experiment are being analyzed, while further measurements are on the schedules of JLab (HAPPEX) and of MAMI (PVA4). Extrapolating the forward angle results towards zero four-momentum transfer and zero scattering angle, has provided a factor of five improvement in the knowledge of the neutral weak couplings to the 'up' and 'down' quarks [15] compared to the entries in the PDG Handbook of 2006.

\section{Tests of the Standard Model}

In going to lower and lower four-momentum transfer and small scattering angle in parity violating electron elastic scattering from the proton, the contributions due to the finite size of the proton become smaller and smaller and one is able to measure then the weak charge of the proton, which constitutes the sum of the weak charges of the two 'up' quarks and the 'down' quark. However, the analyzing power becomes zero at zero momentum transfer and therefore optimum values of four-momentum transfer and incident energy need to be sought. A high precision measurement of the parity violating analyzing power determines the value of $\sin ^{2} \theta_{W}$ and consequently the variation of $\sin ^{2} \theta_{W}$ with four-momentum transfer or the 'running' of $\sin ^{2} \theta_{W}$. The Standard Model makes a definitive prediction of the 'running' of $\sin ^{2} \theta_{W}$ taking into account electroweak radiative corrections once the value of $\sin ^{2} \theta_{W}$ at the $Z^{0}$ pole has been reproduced. As with the QED and QCD couplings, $\alpha\left(\mu^{2}\right)$ and $\alpha_{s}\left(\mu^{2}\right)$ (which exhibit screening and anti-screening, respectively), in going to higher and 


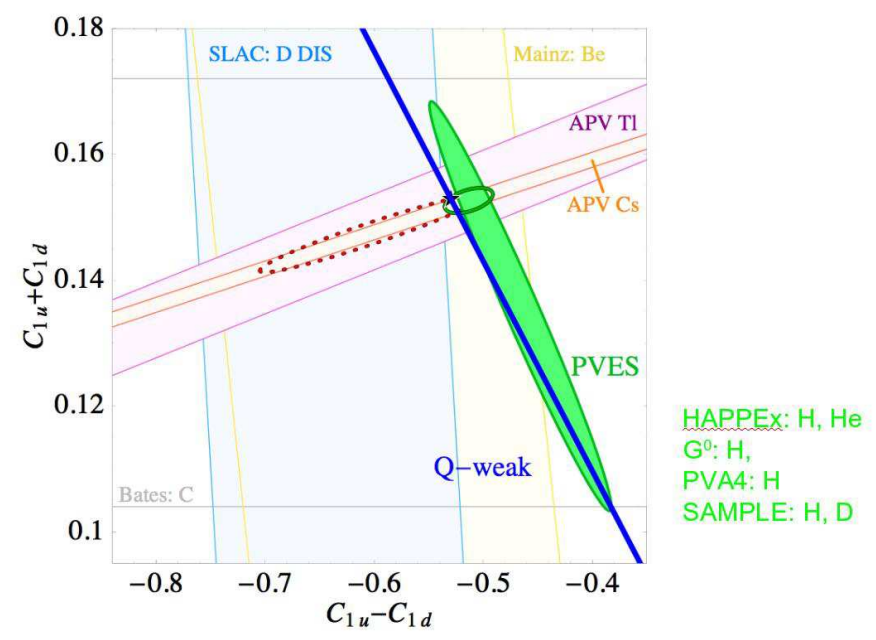

Fig. 5. The neutral weak effective couplings to the quarks. The dotted contour displays the previous experimental limits $(95 \%)$ as reported by the PDG in 2006 together with the prediction of the Standard Model (black star). The filled ellipse gives the new constraint provided by the recent parity violation electron scattering measurements on hydrogen, deuterium, and helium (at 1 standard deviation). The solid contour (95\% CL) presents the constraint based on all experimental results. All other experimental limits shown are displayed at 1 standard deviation. The heavy line represents the anticipated constraint imposed by the Qweak experiment at JLab assuming that the experimental result agrees with the Standard Model.

higher four-momentum transfer, $\sin ^{2} \theta_{W}$ is an effective parameter also varying with $\mu^{2} \approx Q^{2}$. In this case the behaviour with $Q^{2}$ is more subtle since $\sin ^{2} \theta_{W}$ is a function of the electroweak couplings $g_{V l}$ and $g_{A l}:\left(g_{V l} / g_{A l}\right)=1-4 \sin ^{2} \theta_{W}$. Any deviation of $\sin ^{2} \theta_{W}$ from its Standard Model value points to new physics, which needs to be incorporated through a set of new diagrams. In going to very high energies it is plausible that the strong, electromagnetic, and weak interactions become the same. In fact extrapolations to very high energies of the three coupling constants show a 'near-miss' towards having a common intersection (see Fig. 6). Extensions of the Standard Model are therefore in order.

Measurements at the $Z^{0}$ pole have established the value of $\sin ^{2} \theta_{W}$ with great precision although it must be remarked that the leptonic and semi-leptonic values of $\sin ^{2} \theta_{W}$ differ by $3 \sigma$. The Standard Model 'running' of $\sin ^{2} \theta_{W}$ has been calculated by Erler, Kurylov, and Ramsey-Musolf [16] in the modified minimal subtraction scheme (see Fig. 7). The theoretical uncertainties in the 'running' of $\sin ^{2} \theta_{W}$ are represented by the width of the curve. Hence the interpretability is currently limited by the normalization of the curve at the $Z^{0}$ pole, which is arguably as small as \pm 0.00016 . Note the shift of +0.007 at low $Q^{2}$ with respect to the $Z^{0}$ pole best fit value of $0.23113 \pm 0.00015$. There have been reported several low energy measurements of the value of $\sin ^{2} \theta_{W}$. The first one is from an atomic parity violation measurement in Cesium [17], which agrees with the Standard Model 


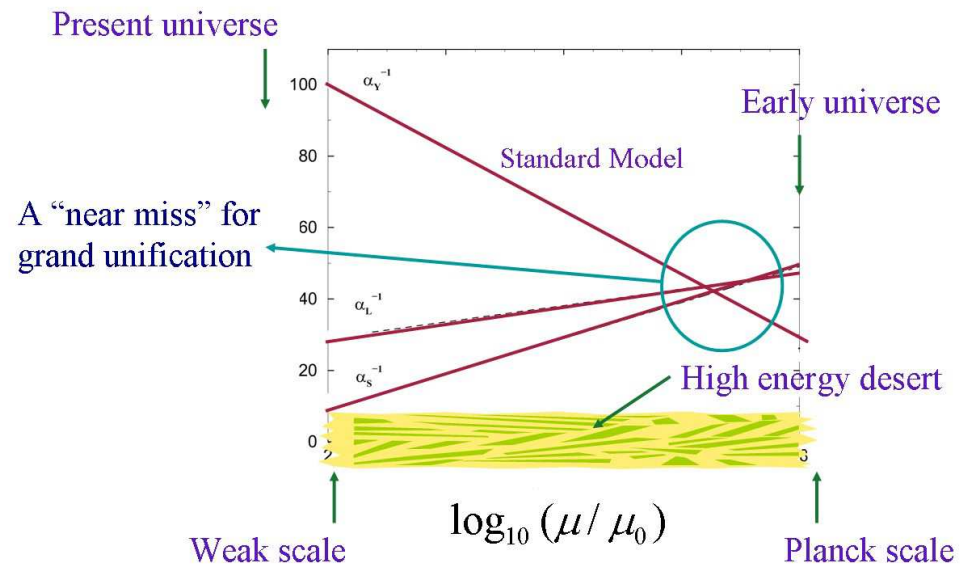

Fig. 6. Extrapolation of the reciprocals of the three coupling constants (electromagnetic, weak, and strong interaction) to the GUT scale

prediction within $1 \sigma$ after many refinements detailing the atomic structure of Cesium were introduced. The second one is from a measurement of parity violating Møller scattering [18], which also agrees with the Standard Model prediction within approximately $1 \sigma$. This is at present the better measurement in constraining extensions of the Standard Model. The third one is from a measurement of neutrino and antineutrino scattering from iron [19] with a roughly $3 \sigma$ deviation from the Standard Model prediction. For this result there remain various uncertainties in the theoretical corrections that need to be applied (among other two distinct effects of charge symmetry breaking in the quark distributions of the nucleons [20]). It is quite apparent that much higher precision experiments are needed in order to search for possible extensions of the Standard Model. One of these is a precision measurement of the weak charge of the proton, $Q_{W}^{p}=1-4 \sin ^{2} \theta_{W}$, currently being prepared for execution starting in 2009 in Hall $\mathrm{C}$ of Jefferson Laboratory [21]. The extraction of the value of $\sin ^{2} \theta_{W}$ is free of many-body theoretical uncertainties and has the virtue of being able to reach much higher precision (note that at a $Q^{2}$ value of $0.03(\mathrm{GeV} / \mathrm{c})^{2}, 1-4 \sin ^{2} \theta_{W}$ equals 0.07 giving a large boost in the precision that may be obtained). The dominant hydronic effects that must be accounted for in extracting $Q_{W}^{p}$ from the measured analyzing power are contained in form factor contributions which are sufficiently constrained from the recent programs of parity violating electron scattering (at MIT-Bates, JLab, and MAMI) without reliance on theoretical nucleon structure calculations, with the exception possibly of very small two-photon exchange contributions). The Standard Model evolution of $\sin ^{2} \theta_{W}$ corresponds to a 10 standard deviation effect in the planned Qweak experiment at JLab. The Qweak experiment, the first ever precision measurement of the weak charge of the proton and more precise than the existing low energy measurements, 


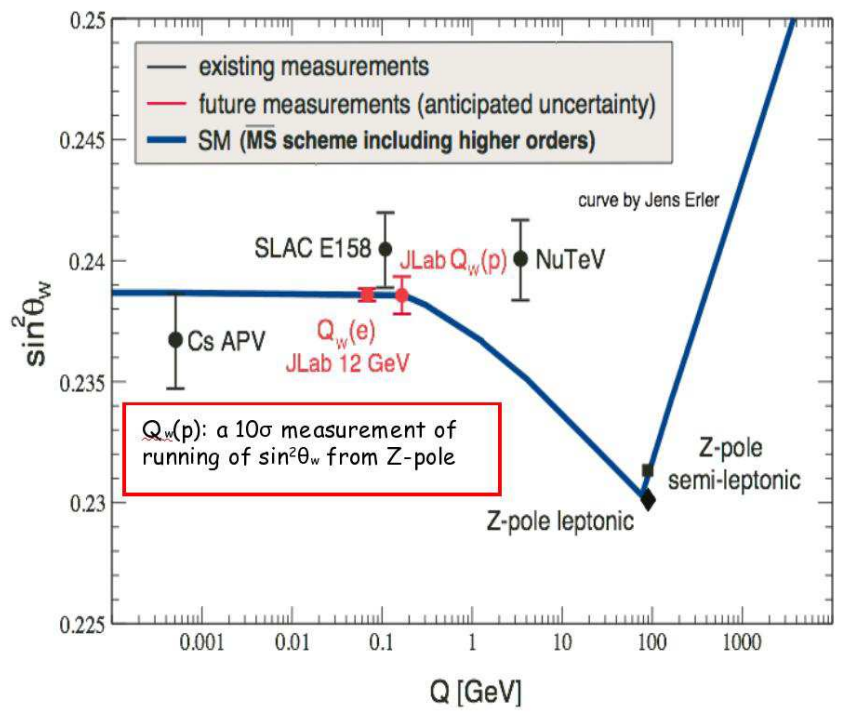

Fig. 7. Calculated 'running' of the weak mixing angle in the Standard Model, as defined in the minimal subtraction scheme. [16] The black points with $1 \sigma$ error bars show the existing experimental values, while the red points with error bars refer to the $4 \% Q_{W}^{p}$ measurement in preparation and a $2.5 \% 1 \mathrm{GeV}$ Møller measurement under consideration.

is crucial in testing the Standard Model.

In the Qweak experiment the weak charge of the proton, $Q_{W}^{p}=1-4 \sin ^{2} \theta_{W}$, will be deduced from the parity violating analyzing power, defined as:

$$
A_{z}=(1 / P)\left[N^{+}-N^{-}\right] /\left[N^{+}+N^{-}\right]
$$

where $\mathrm{P}$ is the polarization of the longitudinally polarized electron beam and $\mathrm{N}^{+}$ and $N^{-}$are the normalized scattering yields. It was shown in [22] that for forward angle scattering, where $\theta \rightarrow 0$, the analyzing power can be written:

$$
A_{z}=\left(-G_{F} /(4 \sqrt{2} \pi \alpha)\right)\left[Q^{2} Q_{W}^{p}+Q^{4} B\left(Q^{2}\right)\right]
$$

Here $G_{F}$ denotes the Fermi coupling constant and $\alpha$ is the fine structure constant. One should note the dependence on $P$, which requires precision polarimetry, and the dependence on the average value of $Q^{2}$ over the finite acceptance of the magnetic spectrometer based detector system for scattered electrons, which requires the averaged value to be determined through specific ancillary control measurements. The leading term in the equation is the weak charge of the proton, $Q_{W}^{p}=1-4 \sin ^{2} \theta_{W}$. The quantity $B\left(Q^{2}\right)$ represents the finite size nucleon structure and contains the proton and neutron electromagnetic and weak form factors. The value of $B\left(Q^{2}\right)$ can be determined experimentally by extrapolation from the ongoing program of forward angle parity violating electron scattering experiments at higher values of $Q^{2}$, discussed above, or by specific control measurements. The incident energy and the four-momentum transfer value (mean scattering angle) followed from careful 


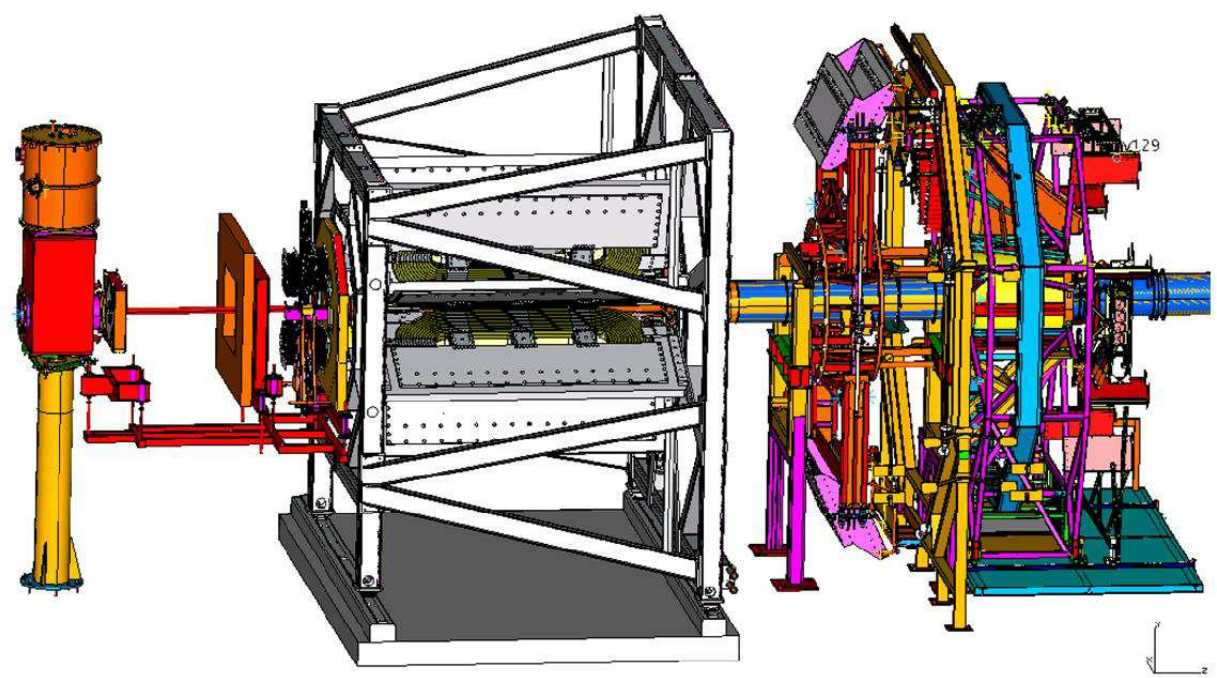

Fig. 8. Layout of the Qweak experiment. The beam is incident from the left and scattered electrons exit the target and pass through the first collimator, the region-1 GEM detectors, the two stage second precision collimator which surrounds the region-2 drift chambers, the toroidal magnet, the shielding wall, the region-3 drift chambers, the trigger scintillators, and finally the ersatz quartz Cerenkov detectors. The tracking system chambers and the trigger scintillators, mounted on rotatable wheels, will be retracted outwards during high current data taking to measure $A_{z}$. The luminosity monitors, which will be used to monitor target density fluctuations and to provide sensitive null tests, are located downstream of the main apparatus very close to the through going beam. Further luminosity monitors are placed just downstream of the $\mathrm{LH}_{2}$ target.

considerations of the figure of merit. The optimum values are an incident energy of $1.165 \mathrm{GeV}$ and a four-momentum transfer of $0.03(\mathrm{GeV} / \mathrm{c})^{2}$. One can then write for the longitudinal analyzing power:

$$
\begin{aligned}
A_{z}\left(0.03(\mathrm{GeV} / \mathrm{c})^{2}\right) & =A\left(Q_{W}^{p}\right)+A\left(\operatorname{Had}_{V}\right)+A\left(\operatorname{Had}_{A}\right) \\
& =-0.19 p p m-0.09 p p m-0.01 p p m
\end{aligned}
$$

where the hadronic structure contributions are separated in vector and axial vector components. Clearly, the analyzing power is very small $(-0.3 p p m)$ and one must arrive at an overall uncertainty of $2 \%$ to meet the precision objective of $0.3 \%$ in $\sin ^{2} \theta_{W}$. Consequently, high statistics data are a prerequisite requiring high luminosity and high beam polarization, and an integrating low-noise detector system of large acceptance. As indicated above, the longitudinal beam polarization must be precisely known as well as the hadronic structure contribution $B\left(Q^{2}\right)$ to be subtracted from the measured analyzing power $\left(A\left(H_{a d}\right)+A\left(H_{V} d_{A}\right)\right)$ The Qweak experiment requires 2200 hours of data taking of the longitudinal analyzing power $A_{z}$ in elastic electron proton scattering at a momentum transfer of $0.03(\mathrm{GeV} / \mathrm{c})^{2}$ with $180 \mu \mathrm{A}$ of $85 \%$ polarized beam incident on a $0.35 \mathrm{~m}$ long $\mathrm{LH}_{2}$ target to determine $\sin ^{2} \theta_{W}$ at the $0.03 \%$ level at low $Q^{2}$. A layout of the Qweak experiment is 
given in Fig. 8.

The Qweak experiment is complementary to a parity violating electron-electron scattering experiment, under consideration to be performed at $11 \mathrm{GeV}$ with an upgraded CEBAF at JLab, with an envisaged precision in $\sin ^{2} \theta_{W}$ equal to or better than that from any individual measurement at the $Z^{0}$ pole. This implies a considerable reduction in the error of the SLAC Møller experiment. Needless to state: the electroweak radiative corrections to a pure leptonic measurement are more contained. In the search for physics beyond the Standard Model, precision measurements of the weak charge of the proton and of the weak charge of the electron are rather complementary.

\section{Acknowledgement}

The author would like to thank W. Desmond Ramsay for critical reading of the manuscript.

\section{References}

1. N. Tanner, Phys. Rev. 107, 1203 (1957).

2. B. Desplanques, J.F. Donoghue, and B.R. Holstein, Ann. Phys. 124, 449 (1980).

3. V.M. Dubovik and S.V. Zenkin, Ann. Phys. 172, 100 (1986).

4. G.B. Feldman, G.A. Crawford, J. Dubach, B.R. Holstein, Phys. Rev. C43, 863 (1991).

5. W.C. Haxton, C.-P. Liu, M.J. Ramsey-Musolf, Phys. Rev. C65, 045502 (2002).

6. P.D. Eversheim, et al., Phys. Lett. B256, 11 (1991).

7. S. Kistryn et al., Phys. Rev. Lett. 58, 1616 (1987).

8. A.R. Berdoz et al. (TRIUMF E497 Collaboration), Phys. Rev. C68, 034004 (2003).

9. J. Carlson et al., Phys. Rev. C65, 035502 (2002).

10. M.J. Ramsey-Musolf and S.A. Page, Ann. Rev. Nucl. Part. Sci. 56, 1 (2006).

11. T.M. Ito et al., (SAMPLE Collaboration), Phys. Rev. Lett. 92, 102003 (2004); D.T. Spayde et al., (SAMPLE Collaboration), Phys. Lett., B583, 79 (2004).

12. K.A. Aniol et al., (HAPPEX Collaboration), Phys. Rev. Lett., 96, 022003 (2006); K.A. Aniol et al., (HAPPEX Collaboration), Phys. Lett., B635, 275 (2006); A. Acha et al., (HAPPEX Collaboration), Phys. Rev. Lett., 98, 032301 (2007).

13. D.S. Armstrong et al., (G0 Collaboration), Phys. Rev. Lett., 95, 092001 (2005).

14. F.E. Maas et al., (PVA4 Collaboration), Phys. Rev. Lett., 93, 022002 (2004); F.E. Maas et al., (PVA4 Collaboration), Phys. Rev. Lett., 94, 152001 (2005).

15. R.D. Young et al., Phys. Rev. Lett., 99, 122003 (2007).

16. J. Erler, A. Kurylov, and M.J. Ramsey-Musolf, Phys. Rev., D68, 016006 (2003).

17. S.C. Bennett and C.E. Wieman, Phys. Rev. Lett., 82, 2484 (1999); C.S. Wood et al., Science, 275, 1759 (1997).

18. P.L. Anthony et al., (SLAC E158 Collaboration),Phys. Rev. Lett., 95, 081601 (2005).

19. G.P. Zeller et al., (NuTeV Collaboration), Phys. Rev. Lett., 88, 091802 (2002).

20. J.T. Londergan, hep-ph/0408243 Nucl. Phys. Proc. Suppl. 141, 68 (2005).

21. R.D. Carlini et al., The Qweak Experiment, JLab Proposal 08-016, Thomas Jefferson National Accelerator Laboratory.

22. M.J. Musolf et al., Phys. Rep., 239, 1 (1994). 